O'Leary, T, Collett, J, Howells, K and Morris, M

Endurance capacity and neuromuscular fatigue following high vs moderate-intensity endurance training: a randomised trial

O'Leary, T, Collett, J, Howells, K and Morris, M (2017) Endurance capacity and neuromuscular fatigue following high vs moderate-intensity endurance training: a randomised trial. Scandinavian Journal of Medicine and Science in Sports

doi: $10.1111 /$ sms.12854

This version is available: https://radar.brookes.ac.uk/radar/items/cced01be-ab00-49cf-af4a-db30025ef829/1/

Available on RADAR: February 2017

Copyright $(C$ and Moral Rights are retained by the author(s) and/ or other copyright owners. A copy can be downloaded for personal non-commercial research or study, without prior permission or charge. This item cannot be reproduced or quoted extensively from without first obtaining permission in writing from the copyright holder(s). The content must not be changed in any way or sold commercially in any format or medium without the formal permission of the copyright holders.

This document is the postprint version of the journal article. Some differences between the published version and this version may remain and you are advised to consult the published version if you wish to cite from it. 


\title{
Endurance capacity and neuromuscular fatigue following high vs moderate-intensity endurance training: a randomised trial
}

\author{
Thomas J O’Leary ${ }^{1}$, Johnny Collett ${ }^{1}$, Ken Howells ${ }^{1}$, Martyn G Morris ${ }^{1,2}$ \\ ${ }^{1}$ Department of Sport and Health Sciences, Oxford Brookes University, Oxford, UK \\ ${ }^{2}$ School of Life Sciences, Coventry University, Coventry, UK
}

\section{Corresponding author}

Thomas J O'Leary

Department of Sport and Health Sciences

Oxford Brookes University

Gipsy Lane

Oxford

OX3 0BP

United Kingdom

Tel: $+44(0) 1865483272$

Email: thomasoleary1988@gmail.com

Running Head: Endurance training and neuromuscular fatigue

Author Contributions: The study was designed by TJO, MGM and JC; TJO and MGM collected and analysed the data; all authors contributed to preparation of the manuscript and approved the final version. 


\section{Abstract}

High-intensity exercise induces significant central and peripheral fatigue, however the effect of endurance training on these mechanisms of fatigue is poorly understood. We compared the effect of cycling endurance training of disparate intensities on high-intensity exercise endurance capacity and the associated limiting central and peripheral fatigue mechanisms. Twenty adults were randomly assigned to 6 weeks of either high-intensity interval training (HIIT, 6-8 $\times 5$ min at halfway between lactate threshold and maximal oxygen uptake $[50 \% \Delta]$ ) or volume matched moderate-intensity continuous training (CONT, $\sim 60-80 \mathrm{~min}$ at $90 \%$ lactate threshold). Two time to exhaustion (TTE) trials at $50 \% \Delta$ were completed preand post-training to assess endurance capacity; the two post-training trials were completed at the pre-training $50 \% \Delta$ (same absolute intensity) and the 'new' post-training $50 \% \Delta$ (same relative intensity). Pre- and post-exercise responses to femoral nerve and motor cortex stimulation were examined to determine peripheral and central fatigue, respectively. HIIT resulted in greater increases in TTE at the same absolute and relative intensities as pretraining ( $148 \%$ and $43 \%$, respectively) compared with CONT ( $38 \%$ and $-4 \%$, respectively). Compared with pre-training, HIIT increased the level of potentiated quadriceps twitch reduction ( $-34 \%$ vs $-43 \%$, respectively) and attenuated the level of voluntary activation reduction ( $-7 \%$ vs $-3 \%$, respectively) following the TTE trial at the same relative intensity. There were no other training effects on neuromuscular fatigue development. This suggests that central fatigue resistance contributes to enhanced high-intensity exercise endurance capacity after HIIT by allowing greater performance to be extruded from the muscle.

Key words: Central nervous system, exercise tolerance, high-intensity interval training, muscle fatigue, transcranial magnetic stimulation. 


\section{Introduction}

High-intensity locomotor exercise induces significant muscle fatigue with mechanisms both central and peripheral in origin (Decorte et al., 2012; O'Leary et al.. 2016). Muscle fatigue is defined as a reduction in maximal force-producing capacity and occurs due to processes distal to the neuromuscular junction (peripheral fatigue) and within the central nervous system (CNS, central fatigue) (Gandevia, 2001). Central fatigue can be measured by stimulation of the motor nerve using the interpolated twitch technique (Merton, 1954). A reduction in torque produced from the resting potentiated muscle twitch during the interpolated twitch technique can also be used to quantify peripheral fatigue. Part of the central component of fatigue arises from supraspinal mechanisms (Sidhu et al., 2009; Goodall et al., 2012) and supraspinal fatigue can be measured with transcranial magnetic stimulation (TMS) of the motor cortex which demonstrates an impairment in the ability to generate maximal motor cortical output (Goodall et al., 2009; Sidhu et al., 2009). Whilst the degree by which central and peripheral fatigue limit endurance capacity are influenced by exercise intensity and duration (Decorte et al., 2012; Thomas et al., 2015; O'Leary et al., 2016; Thomas et al., 2016), and environmental conditions (Goodall et al., 2012; Goodall et al., 2015), the effect of endurance training has yet to be addressed. By investigating the fatigue adaptations induced by endurance training, the processes that allow greater endurance capacity can be better understood to provide further insight into the limiting factors to human endurance.

High-intensity interval training (HIIT) involves repeated bouts of high-intensity exercise, typically $\geq 80 \% \mathrm{HR}_{\max }$, interspersed with brief periods of rest or low-intensity exercise (MacInnis \& Gibala, 2016). HIIT is more effective at improving high-intensity exercise endurance capacity than moderate-intensity continuous training (CONT) (Daussin et al., 
2008; Seiler et al., 2013). Endurance capacity is measured as the time for which a predetermined exercise intensity can be sustained until volitional exhaustion (Burgomaster et al., 2005; Daussin et al., 2008; Seiler et al., 2013). When matched for work completed, HIIT and CONT produce similar improvements in markers of aerobic fitness (lactate threshold [LT] and maximal rate of oxygen uptake [ $\left.\dot{\mathrm{VO}}_{2 \mathrm{max}}\right]$ ) (Poole \& Gaesser, 1985; Edge et al., 2006), suggesting other mechanisms contribute to the greater increase in endurance capacity following HIIT. To date, the majority of studies have examined endurance capacity at workloads prescribed according to pre-training markers of aerobic fitness which are then held constant for the post-training assessment (Burgomaster et al., 2005; Daussin et al., 2008; Seiler et al., 2013). The post-training test is therefore at a lower relative intensity than pretraining due to the increases in aerobic fitness. It is unknown whether the increase in endurance capacity induced by HIIT, is in part, through increasing the ability to tolerate the physiological disturbances induced by high-intensity exercise completed at the same relative intensity as pre-training. The initial focus of this study is to identify whether in an untrained cohort, training under the high physiological strain induced by HIIT increases endurance capacity at the same relative exercise intensity as pre-training.

HIIT may induce greater increases in the muscle oxidative capacity (Daussin et al., 2008) and in vitro muscle buffer capacity (Edge et al., 2006) than CONT. Improved muscle function reduces the metabolic disturbance induced by exercise and attenuates peripheral-fatigue development (Jacobs et al., 2013; Zghal et al., 2015). However, Morales-Alamo et al., (2015) recently demonstrated that the muscle metabolic disturbance is not a limiting factor to wholebody locomotor exercise and a peripheral muscular reserve exists. These authors also demonstrated that the exercising muscle can tolerate a greater degree of metabolic disturbance without harming performance, thus supporting a central limitation to human 
endurance (Morales-Alamo et al., 2015; Torres-Peralta et al., 2015). Endurance training may also induce CNS adaptations that enhance the ability to maintain the central motor drive necessary to continue exercise (Nybo \& Secher, 2004). The development of peripheral fatigue during high-intensity exercise is hypothesised to have an inhibitory effect on central motor drive mediated through the increased firing of group III/IV muscle afferents (Amann, 2011; Amann et al., 2015). Zghal et al., (2015) suggested that endurance training may increase the resistance of the CNS to this inhibitory group III/IV muscle afferent firing. These authors demonstrated that low force knee extensor endurance training increased the level of peripheral fatigue following an exhaustive isometric endurance task without changing central fatigue. However, whilst some evidence exists for the role of group III/IV afferent firing in the development of central fatigue (Gandevia et al. 1996; Sidhu et al., 2014; Sidhu et al. 2017), a recent study does not support a role of group III/IV afferent firing on limiting maximal sprint power following incremental cycling to exhaustion (Torres-Peralta et al., 2015). The role of group III/IV afferents in limiting human endurance therefore remains unclear. Nevertheless, central mechanisms are considered important in exhaustion (Decorte et al., 2012; Morales-Alamo et al., 2015; Torres-Peralta et al., 2015; O'Leary et al., 2016) and it is unknown whether central fatigability adapts in response to whole-body endurance training, and whether there is a difference between HIIT and CONT where the induced level of central and peripheral fatigue is different (O'Leary et al., 2016).

The aim of this study was to compare the effect of HIIT and volume-matched CONT on highintensity exercise endurance capacity and the associated mechanisms of neuromuscular fatigue. It was hypothesised that although aerobic fitness would increase similarly in both groups: i) both HIIT and CONT would increase endurance capacity at the same absolute work rate, but HIIT would increase it to a greater extent; ii) this increase in endurance 
capacity would be accompanied by a reduction in peripheral fatigue at exhaustion but central fatigue would be unchanged; iii) only HIIT would increase endurance capacity at the same relative intensity as pre-training, and; iv) this increase in endurance capacity would be accompanied by an increase in peripheral fatigue at exhaustion, but central fatigue would be unchanged.

\section{Methods}

\section{Participants}

Twenty healthy adults (four females) volunteered to participate in this two-arm parallel-group single-blind randomised study. The study was approved by the institutional ethics review board and carried out in accordance with the Declaration of Helsinki. Participants were recruited in Oxford, UK via advertisements and word of mouth. Each participant completed health history questionnaires and provided written informed consent. All participants were untrained, defined as not currently engaged in structured endurance training $(\leq 30 \mathrm{~min}$ per session, $\leq 2$ sessions per week) as confirmed by initial interview, non-smokers and not taking any medications. After completing the pre-training experimental trials, participants were allocated the next available study number by the blinded assessor. The number related to a computer-generated stratified block randomisation list that randomised individuals $(1: 1)$ into either a HIIT group $(n=10$ [ 8 men $/ 2$ women], age $27 \pm 6$ years, stature $1.75 \pm 0.10 \mathrm{~m}$, mass $79.0 \pm 13.2 \mathrm{~kg}, \dot{\mathrm{V}} \mathrm{O}_{2 \max } 44.5 \pm 5.4 \mathrm{ml} \cdot \mathrm{kg}^{-1} \cdot \mathrm{min}^{-1}$ ) or a group completing work-matched CONT $(\mathrm{n}=10$ [8 men / 2 women], age $27 \pm 4$ years, stature $1.76 \pm 0.11 \mathrm{~m}$, mass $75.4 \pm 12.9$ $\mathrm{kg}, \dot{\mathrm{V}} \mathrm{O}_{2 \max } 43.5 \pm 5.9 \mathrm{ml} \cdot \mathrm{kg}^{-1} \cdot \mathrm{min}^{-1}$ ) based on the $\mathrm{LT}$ and $\dot{\mathrm{V}} \mathrm{O}_{2 \max }$. The list was held by a second investigator not involved in the experimental trials who informed participants and supervised the intervention. Whilst participants could not be blinded, they were naïve to the purpose of the study. Group allocation was concealed from the assessor until the end of the 
study and participants were instructed to refrain from discussing their training programme. All experimental trials were therefore completed by a blinded assessor.

\section{Experimental Procedures}

Participants completed three experimental trials each separated by $48-72 \mathrm{~h}$, before and after 6 weeks of either HIIT or CONT (Fig. 1). All trials were completed at the Movement Science Laboratory at Oxford Brookes University and performed at the same time of day, $2 \mathrm{~h}$ postprandial and after abstaining from caffeine (12 h), alcohol (24 h) and exhaustive exercise (48 h). During the first visit participants completed a submaximal exercise test for the determination of the LT and lactate turn-point (LTP), followed by a maximal exercise test to determine $\dot{\mathrm{V}} \mathrm{O}_{2 \max }$. During the second visit participants underwent the neuromuscular function assessment procedures before completing an endurance capacity (time to exhaustion [TTE]) cycle test at an intensity halfway between $\mathrm{LT}$ and $\dot{\mathrm{VO}}_{2 \max }(50 \% \Delta)$ which served as a familiarisation trial. During the third visit participants repeated the endurance capacity test with neuromuscular function tested pre- and post-exercise to examine central and peripheral fatigue. The training was initiated 2 - $5 \mathrm{~d}$ after the third baseline experimental trial. At followup, the three experimental trials were repeated within $2-4 \mathrm{~d}$ of completing training, however the familiarisation trial was replaced by an endurance capacity trial at the pre-training $50 \% \Delta$ (same absolute intensity [post-abs]) whilst the second endurance capacity trial was completed at the post-training $50 \% \Delta$ (same relative intensity [post-rel]). This allowed a comparison of endurance capacity and fatigue mechanisms at the same absolute work rate as well as the same relative intensity as pre-training. 


\section{Muscle Torque and Electromyography}

Isometric torque $(\mathrm{N} \cdot \mathrm{m})$ produced from the right knee extensors during voluntary and stimulated contractions was measured with participants sitting on an adjustable custom-built strength chair. The hip and knee were flexed at $90^{\circ}$ and the foot placed behind a padded resistance lever that was attached to a four strain-gauge bridge torque transducer. The transducer was integrated into the strength chair at the lever's centre of rotation for the measurement of rotational torque. The lever length was adjusted so the resistance pad was $\sim 2$ $\mathrm{cm}$ above the malleolus and the lever's centre of rotation were aligned to the centre of the knee joint. Straps secured the shoulders and waist to prevent movement, and the chair characteristics were recorded for subsequent replication. The electrical output from the transducer were digitised at a sampling rate of $1000 \mathrm{HZ}$ (Micro $1401 \mathrm{mk}$-II, CED, UK) and recorded on a PC (Spike 2 v5.2, CED, UK). Torque was displayed on a computer screen in front of the chair to assist in providing maximal effort during maximal voluntary contractions (MVC) as well as displaying the target torque for submaximal contractions. The reliability of this apparatus has previously been reported (O’Leary et al., 2016). Electromyography (EMG) was recorded using self-adhesive bipolar $\mathrm{Ag}-\mathrm{AgCl}$ electrodes $(10 \mathrm{~mm}$ diameter, $2 \mathrm{~cm}$ interelectrode distance) placed on the belly of the vastus lateralis and biceps femoris. The areas were shaved and cleaned and the electrodes were photographed to ensure accurate relocation. The EMG signal was amplified $(\times 1000$, NL844, Neurolog, Digitimer, UK), band-pass filtered (10 - $2000 \mathrm{~Hz}$, NL135, Neurolog, Digitimer, UK) and digitised at a sampling rate of $2000 \mathrm{~Hz}$ (Micro $1401 \mathrm{mk}-\mathrm{II}, \mathrm{CED}, \mathrm{UK}$ ). EMG data were collected on PC for subsequent analysis (Spike 2 v.5.2, CED, UK). 


\section{Motor Nerve Stimulation}

Motor nerve stimulation (MNS) was applied at the right femoral nerve using two connecting monophasic magnetic stimulators (BiStim², MagStim, UK) discharged through a 55-mm double branding iron coil (maximum output 2.2T) (Goodall et al., 2009; Amann, 2011; Decorte et al., 2012; O'Leary et al., 2016). Single stimuli were administered from the dual stimulator with the junction of the coil positioned over the nerve, high in the femoral triangle. The site evoking the largest unpotentiated twitch torque $\left(\mathrm{Q}_{\mathrm{tw}, \mathrm{unpot}}\right)$ and EMG response (Mwave amplitude) was determined and marked on the skin with ink. Each participant had a recruitment curve constructed from $\mathrm{Q}_{\mathrm{tw} \text {,unpot }}$ torque and $\mathrm{M}$-wave amplitude determined from two stimuli at $50 \%, 60 \%, 70 \%, 80 \%, 90 \%, 95 \%$ and $100 \%$ of maximum stimulator output. During the pre-training endurance capacity trial, the stimulus-response curve revealed an increment in $\mathrm{Q}_{\mathrm{tw} \text {,unpot }}$ and $\mathrm{M}$-wave amplitude from 90 to $95 \%$ of maximum stimulator output of $1.0 \%(\mathrm{P}=0.29)$ and $0.3 \%(\mathrm{P}=0.48)$, respectively; the increment in $\mathrm{Q}_{\mathrm{tw} \text {,unpot }}$ and $\mathrm{M}$-wave amplitude from $95 \%$ to $100 \%$ of maximum stimulator output was $-0.1 \%(\mathrm{P}=0.88)$ and $-0.2 \%(\mathrm{P}=0.71)$, respectively. During the post-abs trial, the increment in $\mathrm{Q}_{\mathrm{tw}, \text { unpot }}$ and $\mathrm{M}-$ wave amplitude from 90 to $95 \%$ of maximum stimulator output was $0.6 \%(\mathrm{P}=0.71)$ and $1.4 \%(\mathrm{P}=0.30)$, respectively; the increment in $\mathrm{Q}_{\mathrm{tw} \text {,unpot }}$ and $\mathrm{M}$-wave amplitude from $95 \%$ to $100 \%$ of maximum stimulator output was $-0.6 \%(\mathrm{P}=0.66)$ and $-0.2 \%(\mathrm{P}=0.81)$, respectively. Thus, a plateau in torque and M-wave amplitude was confirmed at both the preand post-training time-points in all participants. All remaining stimulations were given at $100 \%$ of maximal stimulator output.

\section{Transcranial Magnetic Stimulation}

Transcranial magnetic stimulation (TMS) was performed over the left motor cortex in order to activate the right vastus lateralis as previously completed in our laboratory (O'Leary et al., 
2015; O'Leary et al., 2016). Two connecting monophasic magnetic stimulators (BiStim ${ }^{2}$, MagStim, UK) were discharged through a 110-mm double cone coil (maximum output 1.4T). The coil was held above the vertex and orientated to induce an intracranial posterior to anterior current. The optimal location for the vastus lateralis was determined with the stimulator output set at $60 \%$ of maximum. The vertex of the scalp was located and the search for the optimal location began with the coil placed $1 \mathrm{~cm}$ lateral to the vertex over the left hemisphere. The coil was moved in $1 \mathrm{~cm}$ steps in the lateral-medial and anterior-posterior directions until the area of stimulation that elicited the largest motor evoked potential (MEP) during a $10 \%$ MVC was identified. This site was marked using permanent marker on the scalp to ensure accurate relocation of the coil within session. Resting motor threshold (rMT) was determined by reducing the stimulator output until the lowest intensity that elicited a minimum of $50 \mu \mathrm{V}$ in 5 out of 10 pulses was determined (Chen et al., 2008). The rMT was determined to the nearest $1 \%$ of maximum stimulator output at pre-exercise during each visit and used for setting the TMS intensities which were held then constant within that trial. The rMT for the pre-training, post-abs and post-rel trials equated to $51 \pm 10 \%, 51 \pm 10 \%$ and $53 \pm$ $10 \%$, respectively, for the HIIT group, and $47 \pm 9 \%, 46 \pm 8 \%$, and $46 \pm 9 \%$, respectively, for the CONT group. The rMT was not different across trials $(\mathrm{P}>0.05)$.

\section{Neuromuscular Function}

In order to examine the effect of each endurance capacity test on central and peripheral fatigue, pre- to post-exercise changes in voluntary, MNS and TMS evoked torque and EMG responses were determined (Fig. 1b). At the beginning of each visit a series of familiarisation MVCs were performed until torque plateaued. Three MVCs were then performed with MNS applied at peak torque and $\sim 3 \mathrm{~s}$ post MVC to determine voluntary activation (VA), potentiated twitch torque $\left(\mathrm{Q}_{\mathrm{tw}, \mathrm{pot}}\right)$ and maximal $\mathrm{M}$-wave amplitude (resting $\left[\mathrm{M}_{\max }\right]$ and during 
the MVC $\left.\left[\mathrm{M}_{\text {sup }}\right]\right)$. Each MVC was held for $3 \mathrm{~s}$ with $15 \mathrm{~s}$ separating each effort (Decorte et al., 2012). The peak of the three MVCs was taken as peak torque and used to determine the target torque for the remaining submaximal contractions at that time point. A set of three contractions at $100 \%, 75 \%$ and $50 \%$ MVC were then completed with single TMS pulses at $130 \%$ rMT administered during each contraction for the determination of cortical voluntary activation (cortical VA) (Goodall et al., 2009). Each contraction was separated by $\sim 3-5 \mathrm{~s}$ and each set was repeated three times separated by $15 \mathrm{~s}$ (Goodall et al., 2012; Thomas et al., 2015). The TMS intensity elicited a large MEP in the vastus lateralis during a $50 \% \mathrm{MVC}$ (mean amplitude of $63 \pm 26 \% \mathrm{M}_{\text {sup }}$ ) and small MEP in the biceps femoris (mean amplitude of $7 \pm 3 \%$ of vastus lateralis $M_{\text {sup }}$ ), data grouped for both groups and all time-points. We have previously shown the reliability of these procedures for the measurement of neuromuscular fatigue (MVC, $\mathrm{Q}_{\mathrm{tw}, \mathrm{pot}}$, VA and cortical VA loss) after high-intensity exhaustive cycling to be moderate to good (ICC $=0.62-0.88)\left(\mathrm{O}^{\prime}\right.$ Leary et al., 2016).

\section{Exercise Tests}

Exercise tests were completed on an electromagnetically braked cycle ergometer (Excalibur Sport, Lode, Netherlands) at a self-selected cadence above $60 \mathrm{rpm}$. During the first visit, participants adjusted the seat height and handle bar distance to ensure a comfortable position and the cycle set-up was recorded to allow replication. Heart rate (HR) was recorded using online telemetry (T31, Polar, Finland) and expired gases were recorded breath-by-breath using an online gas analyser (Metalyzer 3B, Cortex, Germany) for the determination of rate of oxygen uptake $\left(\dot{\mathrm{VO}}_{2}\right)$ and respiratory exchange ratio (RER). Blood lactate concentration ([[a] $)$ was measured from a whole blood finger-tip capillary sample (Lactate Pro, Arkray, Japan) and ratings of perceived exertion (RPE) were determined using the Borg Scale (6 20). 


\section{Aerobic Fitness}

Participants first completed a submaximal exercise test for the determination of the LT and LTP. The submaximal test consisted of cycling at a starting power of $50 \mathrm{~W}$ for males and 30 W for females with the power output increased by $20 \mathrm{~W}$ every $4 \mathrm{~min}$. [La'] was measured at the end of each stage and the test was terminated once [La-] reached $\geq 4 \mathrm{mmol} \cdot \mathrm{l}^{-1}$. The LT was defined as the first sudden and sustained increase in [ $\left.\mathrm{La}^{-}\right]$and the LTP was defined as the second sudden increase in $\left[\mathrm{La}^{-}\right]$between the LT and $\dot{\mathrm{V}}{ }_{2 \max }$, both confirmed by visual inspection of the power output and $\left[\mathrm{La}^{-}\right]$relationship by two blinded reviewers. If the two reviewers disagreed, a third reviewer was consulted. A maximal exercise test was completed 20 min after the completion of the submaximal test for the determination of $\dot{\mathrm{VO}}_{2 \max }$. The maximal test began with cycling for $1 \mathrm{~min}$ at $100 \mathrm{~W}$ for males and $50 \mathrm{~W}$ for females followed by a ramp increase of $25 \mathrm{~W} \cdot \mathrm{min}^{-1}$ for males and $20 \mathrm{~W} \cdot \mathrm{min}^{-1}$ for females until $60 \mathrm{rpm}$ could no longer be maintained. $\dot{\mathrm{V}} \mathrm{O}_{2 \max }$ was taken as the highest $30 \mathrm{~s}$ average of the recorded $\dot{\mathrm{V}} \mathrm{O}_{2}$. Power output at $\dot{\mathrm{V}} \mathrm{O}_{2 \max }$ was extrapolated from the linear relationship between $\dot{\mathrm{V}} \mathrm{O}_{2}$ and power output from sub-LT intensities and was used in the calculation of $50 \% \Delta$.

\section{Endurance Capacity}

High-intensity exercise endurance capacity was examined with a series of TTE trials. All trials were preceded by a 5 min self-paced warm-up completed below the LT. The test was terminated if a cadence of $60 \mathrm{rpm}$ could no longer be maintained for $5 \mathrm{~s}$ or if the participant fell below this cadence three times. Participants were blinded to elapsed time and encouraged to continue as long as possible. The neuromuscular fatigue testing protocol was performed before exercise and repeated as soon as possible post-exhaustion. There was no difference between groups or trials for the time of measurement post-exercise $(51 \pm 20 \mathrm{~s}$ for HIIT and 
$53 \pm 20 \mathrm{~s}$ for CONT, data for all trials grouped) or duration of neuromuscular assessment $(163 \pm 17 \mathrm{~s}$ for HIIT and $160 \pm 24 \mathrm{~s}$ for CONT, data for all trials grouped) $(\mathrm{P} \geq 0.86)$. Expired gases, HR, RPE and [ $\left.\mathrm{La}^{-}\right]$were recorded pre-exercise, after $10 \mathrm{~min}$ of exercise and during the final min of exercise. The trials were completed at $50 \% \Delta$ in order to target an intensity above maximal lactate steady-state (Pringle \& Jones, 2002) and to induce significant central and peripheral fatigue (O'Leary et al., 2016). Prescription of intensity relative to metabolic thresholds ensures comparable metabolic and perceptual responses between participants (Mann et al., 2013). The $50 \% \Delta$ was recalculated after training so the post-training TTE trials could be completed at both the pre-training $50 \% \Delta$ (post-abs) and post-training $50 \% \Delta$ (postrel) (Fig. 1a).

\section{Training}

All training was completed at the Human Performance Laboratory at Oxford Brookes University on a cycle ergometer (Excalibur Sport or Corival, Lode, Netherlands) and was supervised by an investigator not involved in the experimental trials. Both the HIIT and CONT groups completed 18 sessions over 6 weeks $\left(3 \cdot \mathrm{wk}^{-1}\right)$ with each session separated by at least $24 \mathrm{~h}$. During training participants were instructed to maintain normal habitual activity levels. An adapted HIIT protocol was used (Weston et al., 1997) which involved 6 repeats of 5 min cycling at $50 \% \Delta$, each separated by 1 min rest, which was progressed to 8 repeats during weeks 4-6. The CONT protocol involved completing the same volume of work (kJ) that would be completed in the HIIT protocol at $90 \% \mathrm{LT}$. Training near the LT provides an aerobic stimulus but prevents the non-linear increase in metabolic and perceptual stress seen with supra-LT exercise (Jones \& Carter, 2000). Training intensities were prescribed relative to metabolic thresholds in order to minimise inter-individual responses to the training and to elicit disparate metabolic and perceptual demands between groups (Mann et al., 2013). 
During each training session, HR and RPE were measured after every 5 min of exercise. Intensity was re-assessed every 2 weeks (session 7 and 13) and increased if necessary to ensure HR and RPE were consistent throughout training.

\section{Data Analysis}

All analyses were completed by a blinded investigator. MVC torque was taken as the peak of three contractions and $\mathrm{Q}_{\mathrm{tw}, \text { pot }}, \mathrm{M}_{\max }, \mathrm{VA}$ and cortical VA were taken as the mean of the three measures at each time point. Superimposed twitch torque in response to MNS and TMS were calculated by subtracting twitch onset torque from peak twitch torque. VA measured with MNS was calculated using the formula: [1 - (superimposed twitch torque / $\left.\left.\mathrm{Q}_{\mathrm{tw}, \mathrm{pot}}\right) \times 100\right]$. For the determination of cortical VA, the resting twitch was estimated (ERT) from the yintercept of the linear relationship between voluntary and superimposed twitch torque across the contraction intensities of 50\%,75\% and 100\% MVC (Goodall et al., 2009; Sidhu et al., 2009). Regression analysis confirmed the linearity of this relationship $\left(\mathrm{r}^{2}=0.93 \pm 0.05\right.$, mean across both groups and all time-points). Cortical VA was calculated using the formula: [1 (superimposed twitch / ERT) × 100] (Goodall et al., 2009; Sidhu et al., 2009). For all MNS and TMS stimulations, peak-to-peak amplitude of the EMG responses was calculated.

\section{Statistical Analysis}

Data are mean $\pm \mathrm{SD}$ in the tables and text and mean $\pm \mathrm{SE}$ in the figures. Statistical analyses were completed in SPSS (v.23, SPSS Inc., USA). All data were tested for normality and subsequently investigated with ANOVAs (see below). Where the assumption of sphericity was violated, Greenhouse-Geisser corrections were applied. Where an ANOVA revealed a significant main effect or interaction, post-hoc contrasts and t-tests were used to test for differences within and between groups where appropriate. Effect sizes were calculated with 
partial eta squared $\left(\eta_{\mathrm{p}}{ }^{2}\right)$ where an ANOVA design was used (interaction and main effects) and Cohen's D where a t-test design was used (within-group pre- to post-training changes or within-trial pre- to post-exercise changes). Significance was accepted as $\mathrm{P} \leq 0.05$.

\section{Training Intensity and Volume, Aerobic Fitness and Endurance Capacity}

Total training volume, and baseline aerobic fitness and endurance capacity were compared between groups with independent samples t-tests. A series of $2 \times 2$ mixed-design ANOVAs was used to compare training interventions for: i) within-training data (group [HIIT and $\mathrm{CONT}] \times$ time $[$ first session and last session]); ii) aerobic fitness (group [HIIT and CONT] $\times$ time [pre-training and post-training]); iii) endurance capacity (group [HIIT and CONT] $\times$ trial [pre-training and post-abs or post-rel]), and; iv) within-exercise data during the endurance capacity trials (group [HIIT and CONT] $\times$ trial [pre-training and post-abs or post-rel]).

\section{Neuromuscular Fatigue}

To compare training protocols on the development of central and peripheral fatigue, measures of neuromuscular function were examined with $2 \times 2 \times 2$ (group [HIIT and CONT] $\times$ trial [pre-training and post-abs or post-rel] $\times$ time (pre-exercise and post-exercise]) mixed-design ANOVAs. Significant group $\times$ trial $\times$ time interactions were followed up with $2 \times 2$ (trial [pre-training and post-abs or post-rel] $\times$ time [pre-exercise and post-exercise]) repeated measure ANOVAs to independently examine the effect of each training protocol on the development of fatigue.

\section{Results}

\section{Training Intensity and Volume}


All training data can be seen in Table 1. All participants successfully completed the training intervention and there was excellent adherence with $98 \%$ of HIIT sessions completed (3 missed) and $99 \%$ of CONT sessions completed ( 2 missed). There were no differences in power output, HR or RPE progression between groups (group $\times$ time interactions, all $\mathrm{P} \geq$ 0.303). Power output, HR and RPE were higher during HIIT compared with CONT (main effects of group, all $\mathrm{P}<0.001)$. Power output was higher during the last session compared with the first session (main effect of time, $\mathrm{P}<0.001$ ), however HR and RPE were similar between sessions (main effects of time, both $\mathrm{P} \geq 0.296$ ). Therefore, the training intensity was adequately increased in both groups to ensure a similar training stimulus throughout. Total training volume was not different between groups $(\mathrm{P}=0.809)$.

\section{Aerobic Fitness}

There were no differences between groups for pre-training measures of LT, LTP or $\dot{\mathrm{V}} \mathrm{O}_{2 \max }$ (all $\mathrm{P} \geq 0.692$ ) or training-induced changes in these measures (group $\times$ time interactions, all $\mathrm{P}$ $\geq 0.207, \eta_{\mathrm{p}}{ }^{2} \leq 0.087$ ) (Table 2). Training increased the power at the LT (HIIT: $24 \pm 20 \%$, CONT: $23 \pm 10 \%$, power at LTP (HIIT: $19 \pm 12 \%$, CONT: $14 \pm 10 \%)$ and $\dot{\mathrm{VO}}{ }_{2 \max }\left(1 \cdot \mathrm{min}^{-1}\right)$ (HIIT: $8 \pm 7 \%$, CONT: $9 \pm 9 \%$ ) (main effects of time, all $\mathrm{P}<0.001, \eta_{\mathrm{p}}{ }^{2} \geq 0.580$ ). The $\dot{\mathrm{V}} \mathrm{O}_{2}$ $\left(\% \dot{\mathrm{VO}}_{2 \max }\right)$ at LT and LTP showed a similar pattern of change. Accordingly, both HIIT and CONT increased the power at $50 \% \Delta$ to a similar extent (Table 2).

\section{Endurance Capacity}

\section{Post-Absolute Trial}

The coefficient of variation for TTE between the familiarisation and pre-training experimental trial was $11.8 \%$. There were no differences between groups for pre-training TTE $(\mathrm{P}=0.282)$. There was a significant group $\times$ trial interaction for TTE $(\mathrm{P}<$ 
0.001, $\left.\eta_{\mathrm{p}}{ }^{2}=0.523\right)$. Post-hoc t-tests revealed that TTE during the post-abs trial was significantly higher than the pre-training trial after HIIT $(148 \pm 74 \%, \mathrm{P}<0.001, \mathrm{D}=2.112)$ and CONT $(38 \pm 52 \%, \mathrm{P}=0.018, \mathrm{D}=0.747)$ with the group $\times$ trial interaction demonstrating the increase was greater after HIIT (Fig. 2). Both HIIT and CONT attenuated some of the within-exercise responses during the post-abs trial compared to pre-training, however there were no differences between groups (Table 3).

\section{Post-Relative Trial}

There was a significant group $\times$ trial interaction for TTE $\left(P=0.019, \eta_{\mathrm{p}}{ }^{2}=0.269\right)$. Post-hoc $\mathrm{t}-$ tests revealed TTE during the post-rel trial was significantly higher than the pre-training trial after HIIT $(43 \pm 56 \%, \mathrm{P}=0.011, \mathrm{D}=0.854)$ but not after CONT $(\mathrm{P}=0.342, \mathrm{D}=0.472)$ with the group $\times$ trial interaction demonstrating the increase was greater after HIIT (Fig. 2). There were no effects of training on the within-exercise responses for either group (Table 3).

\section{Neuromuscular Fatigue}

Figure 3 shows an example raw EMG and torque trace for the measurement of MVC torque,

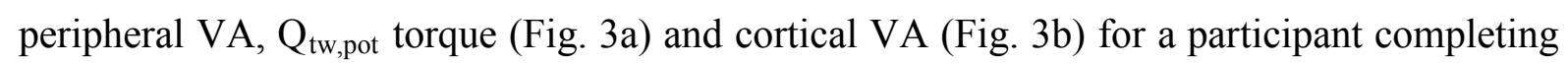
HIIT.

\section{Post-Absolute Trial}

When comparing the post-abs trial to the pre-training trial, neither HIIT or CONT had an effect on the MVC, $\mathrm{Q}_{\mathrm{tw}, \text { pot }}, \mathrm{VA}$ or cortical VA responses to exercise (trial $\times$ time interactions, all $\left.\mathrm{P} \geq 0.052, \eta_{\mathrm{p}}{ }^{2} \leq 0.194\right)$ with no differences between groups (group $\times$ trial $\times$ time interactions, all $\left.\mathrm{P} \geq 0.088, \eta_{\mathrm{p}}{ }^{2} \leq 0.161\right)$. Nevertheless, compared with pre-exercise, $\mathrm{MVC}, \mathrm{Q}_{\mathrm{tw}, \mathrm{pot}}$, VA and cortical VA were all reduced post-exercise in the pre-training and 
post-abs trials for both groups (main effects of time, all $\mathrm{P}<0.001, \eta_{\mathrm{p}}{ }^{2} \geq 0.573$ ) (Fig. 4). There was no effect of HIIT or CONT on the $M_{\max }$ or MEP responses to exercise for any contraction strength (Table 4).

\section{Post-Relative Trial}

When comparing the post-rel trial to the pre-training trial, neither HIIT or CONT had an effect on the MVC or cortical VA response to exercise (trial $\times$ time interactions, both $\mathrm{P} \geq$ $0.114, \eta_{\mathrm{p}}{ }^{2} \leq 0.133$ ) with no differences between groups (group $\times$ trial $\times$ time interactions, both $\left.\mathrm{P} \geq 0.089, \eta_{\mathrm{p}}{ }^{2} \leq 0.173\right)$. Nevertheless, compared with pre-exercise both the MVC and cortical VA were reduced post-exercise in the pre-training and post-rel trials for both groups (main effects of time, both $\mathrm{P} \leq 0.001, \eta_{\mathrm{p}}{ }^{2} \geq 0.518$ ) (Fig. 4a and Fig. 4d). There was a significant group $\times$ trial $\times$ time interaction for measures of $\mathrm{Q}_{\mathrm{tw}, \mathrm{pot}}\left(\mathrm{P}=0.016, \eta_{\mathrm{p}}{ }^{2}=0.298\right)$ and VA $\left(P=0.016, \eta_{\mathrm{p}}{ }^{2}=0.298\right)$. Post-hoc t-tests revealed that the post-exercise $\mathrm{Q}_{\mathrm{tw} \text {,pot }}$ and VA were lower than the corresponding pre-exercise values for both the pre-training and postrel trials in the HIIT group (all $\mathrm{P} \leq 0.024, \mathrm{D} \geq 0.947$ ). HIIT increased the exerciseinduced $\mathrm{Q}_{\mathrm{tw} \text {,pot }}$ reduction (post-rel: $-43 \pm 13 \%$ vs pre-training: $-34 \pm 17 \%$, trial $\times$ time interaction, $\left.\mathrm{P}=0.023, \eta_{\mathrm{p}}{ }^{2}=0.453\right)$ and attenuated the exercise-induced reduction in VA (post-rel: $-3 \pm 3 \%$ vs pre-training: $-7 \pm 7 \%$, trial $\times$ time interaction, $\mathrm{P}=0.047, \eta_{\mathrm{p}}{ }^{2}=0.371$ ), which were both significantly different from CONT (Fig. 4b and Fig. 4c). CONT had no effect on the $\mathrm{Q}_{\mathrm{tw} \text {,pot }}$ or VA reductions following exercise (trial $\times$ time interactions, both $\mathrm{P} \geq$ $0.150, \eta_{\mathrm{p}}{ }^{2} \leq 0.216$ ) (Fig. $4 \mathrm{~b}$ and Fig. $4 \mathrm{c}$ ). There was no effect of HIIT or CONT on the $\mathrm{M}_{\max }$ or MEP responses to exercise for any contraction strength (Table 4).

\section{Discussion}


The aim of this study was to compare the effect of 6 weeks high-intensity interval training and volume-matched moderate-intensity continuous training on high-intensity exercise endurance capacity and the associated mechanisms of neuromuscular fatigue. The main findings are that despite similar improvements in aerobic fitness (LT, LTP and $\dot{\mathrm{V}}_{2 \max }$ ), HIIT resulted in markedly greater improvements in endurance capacity (TTE) when completed at the same power output as pre-training (post-abs trial) compared to CONT. Furthermore, when the post-training endurance capacity test was repeated at the same relative intensity as pretraining (post-rel trial) to account for changes in aerobic fitness, only HIIT improved endurance capacity. This increase in post-rel endurance capacity after HIIT was accompanied by a greater level of peripheral fatigue with attenuated central fatigue. By comparing HIIT to volume-matched CONT in a randomised trial, we can isolate mechanisms specific to HIIT that are independent of improvements in aerobic fitness and any behavioural artefacts associated with completing a training intervention. Consequently, this study provides new insight into the mechanisms underpinning the greater enhancement of high-intensity exercise endurance capacity following HIIT and contributes to the understanding on central and peripheral limitations to endurance capacity.

The training intensities in the present study were prescribed relative to metabolic thresholds recorded during an incremental exercise test. The intensity of the HIIT was set at $50 \% \Delta$ in order to target an intensity above LTP (no steady-state), whereas the CONT intensity was set at $90 \% \mathrm{LT}$ and was therefore in the moderate-intensity domain (steady-state). This was in order to elicit comparable metabolic, perceptual and neuromuscular fatigue responses withingroup and disparate responses between-groups (Tschakert \& Hofmann, 2013; O'Leary et al., 2016). There are however, a variety of training protocols employed in previous research with the classification of intensity for both HIIT and CONT unclear. Previous suggestions for 
defining high-intensity include intervals of exercise $\geq 80 \% \mathrm{HR}_{\max }$ (MacInnis \& Gibala, 2016) or greater than LTP but below the power achieved during an all-out effort (Tschakert \& Hofmann, 2013). These criteria are in line with the intensity employed in our study but results in submaximal (i.e. below $\mathrm{VO}_{2 \max }$ ) intervals. Higher training intensities have been employed including repeated bouts of all-out supramaximal efforts (sprint-interval training) (MacInnis \& Gibala, 2016). Although difficult to match training volume between two training groups when employing low-volume sprint-interval training, it is unclear if our findings translate to supramaximal training intensities and is a potential area for future work.

\section{Endurance Capacity}

When tested at the same power output as pre-training (post-abs trial), TTE was enhanced in both training groups, however the increase was greater following HIIT (147\%) compared with CONT (38\%) in agreement with previous work (Daussin et al., 2008; Seiler et al., 2013). There were no differences between groups for improvements in aerobic fitness (LT, LTP or $\dot{\mathrm{V}} \mathrm{O}_{2 \max }$ ), also in agreement with previous work-matched HIIT and CONT training studies (Poole \& Gaesser, 1985; Edge et al., 2006). Therefore, changes in aerobic fitness cannot explain the differences in endurance capacity between groups. Whilst the better endurance in the post-abs trial following HIIT could be due to greater improvements in mitochondrial function (Daussin et al., 2008) and in vivo muscle buffer capacity (Edge et al., 2006), there is limited understanding regarding how other physiological adaptations compare between HIIT and CONT (MacInnis \& Gibala, 2016).

When the endurance-capacity test was repeated at a power output that was increased to account for the training-induced increases in aerobic fitness (post-rel trial), only HIIT resulted in improvements in TTE ( $43 \%$ vs $-4 \%$ following CONT). In order to better 
understand the mechanisms by which HIIT increases high-intensity exercise endurance capacity versus CONT, the post-training power output was increased so endurance could be compared at the same relative exercise intensity as pre-training. The $\% \Delta$ method allows improvements in both $\mathrm{LT}$ and $\dot{\mathrm{V}} \mathrm{O}_{2 \max }$ to be accounted for and $50 \% \Delta$ was recalculated posttraining to elicit a similar physiological strain to pre-training. Both the pre-training and posttraining 50\% $\Delta$ were above the LTP in all participants and the similar HR, RER, [La-] and RPE responses compared with pre-training demonstrates a similar cardio-respiratory, metabolic and perceptual stress was induced and therefore changes in these parameters cannot explain the enhanced post-rel TTE following HIIT. Demarle et al., (2001) reported that 8 weeks HIIT failed to improve running TTE at the same relative intensity as pretraining, however the participants were trained runners. Whether training enhances the ability to tolerate the same physiological strain was therefore contentious (Millet, 2011) and our study provides the first evidence that in an untrained cohort, HIIT increases high-intensity endurance capacity despite no attenuation of the physiological stress or perception of effort induced by the exercise. The comparison with CONT demonstrates these effects are not due to an increase in the aforementioned parameters of aerobic fitness. This supports our hypothesis that training under high physiological strain and perception of effort improves endurance capacity by increasing the ability to tolerate this strain and effort, compared with training at moderate-intensities where the physiological disturbance and perception of effort are moderate.

\section{Neuromuscular Fatigue}

The endurance capacity trials all induced significant peripheral fatigue, as measured by the $\mathrm{Q}_{\mathrm{tw}, \text { pot }}$ reduction from pre- to post-exercise. The large reduction in $\mathrm{Q}_{\mathrm{tw}, \text { pot }}$ suggests that peripheral fatigue contributed to the majority of the MVC loss. Exercise did not change the 
$\mathrm{M}_{\max }$, in agreement with previous high-intensity locomotor exercise studies (Goodall et al., 2012; Thomas et al., 2016), which suggests that a loss of muscle membrane excitability did not contribute to the peripheral fatigue (Allen et al., 2008). Potential mechanisms include impaired sarcoplasmic reticulum calcium $\left(\mathrm{Ca}^{2+}\right)$ release and uptake, reduced $\mathrm{Ca}^{2+}$ sensitivity of the contractile apparatus and reduced maximum $\mathrm{Ca}^{2+}$ activated force production due to the increase in a number of intramuscular metabolites such as inorganic phosphate (Allen et al., 2008).

In contrast to our hypothesis that HIIT would attenuate peripheral fatigue, the level of peripheral fatigue induced by the post-abs trial was similar to pre-training following both training interventions. This was despite an attenuation of the metabolic stress induced by the exercise as demonstrated by the lower [ $\left.\mathrm{La}^{-}\right]$, RER and HR recorded at isotime (10 min) during the post-abs trials compared to pre-training. Although peripheral fatigue at exhaustion was not different between trials, the volume of work completed during the post-abs trial before achieving this level of peripheral fatigue was far greater than pre-training following HIIT. Exhaustion following constant-load moderate-intensity cycling (O'Leary et al., 2016) and heavy-intensity cycling (Thomas et al., 2016) occurs with a lower level of peripheral fatigue than severe-intensity cycling. It is therefore unclear why HIIT failed to reduce the level of peripheral fatigue at exhaustion despite attenuating the metabolic demands of the exercise, however an increase in peripheral fatigue tolerance (Zghal et al., 2015) may have negated any effect of improved peripheral fatigue resistance.

Compared with pre-training, HIIT increased the level of peripheral fatigue following the post-rel trial, whilst CONT had no effect. Peripheral fatigue is a repeatable measure following cycling to exhaustion at 50\% (O'Leary et al., 2016), strengthening this finding. 
Small increases in the resting $\mathrm{Q}_{\mathrm{tw}, \text { pot }}$ following HIIT may have contributed to this result; although the relative reduction in $\mathrm{Q}_{\mathrm{tw} \text {,pot }}$ was increased, it could also be interpreted that exhaustion occurred at the same level of absolute $\mathrm{Q}_{\mathrm{tw} \text {,pot }}$ torque (Table 4). It is therefore possible that the greater level of peripheral fatigue may be due to a greater muscle reserve above a critical level of absolute $\mathrm{Q}_{\mathrm{tw}, \mathrm{pot}}$ torque, perhaps due to the same motoneuron pool being exhausted. However, small increases in the resting $\mathrm{Q}_{\mathrm{tw} \text {,pot }}$ as a result of training might be expected to also increase the end exercise $\mathrm{Q}_{\mathrm{tw} \text {,pot }}$ (i.e. muscle hypertrophy) and therefore peripheral fatigue would be equal to pre-training. Indeed, this was exactly what we found following CONT. Furthermore, the small increases in the resting $\mathrm{Q}_{\mathrm{tw}, \mathrm{pot}}$ following training were not different between groups $(\mathrm{P}=0.418)$ and we are therefore confident that HIIT independently increased the level of peripheral fatigue. Similarly, isolated knee extensor training has shown to increase the level of peripheral fatigue following a low intensity isometric contraction completed until exhaustion, compared to the corresponding pre-training value (Zghal et al., 2015). However, the control group performed no training and the exercise mode was isolated contractions where the central feedback mechanisms are likely different to locomotor exercise (Sidhu et al., 2013). Therefore, it was previously unclear whether any behavioural artefacts associated with completing training contributed to their findings and whether the effects were transferable to whole-body locomotor exercise. The data here suggest the increase in peripheral fatigue was training intensity dependent and not due to increases in aerobic fitness.

Zghal et al., (2015) suggested that endurance training increases the CNS resistance to the inhibitory actions of group III/IV muscle afferents either through an increase in the sensory threshold of the afferents or via supraspinal adaptations which increases peripheral fatigue tolerance. Peripheral fatigue has been proposed to play an important role in exhaustion 
through group III/IV afferent inhibition of central motor drive (Amann, 2011; Amann et al., 2015). Some evidence exists for a role of group III/IV afferent firing in the development of central fatigue using post-exercise ischemia to maintain group III/IV afferent firing following a 2 min sustained MVC (Gandevia et al., 1996) or pharmacological blockade of the central projection of group III/IV muscle afferents following exhaustive high-intensity cycling (Sidhu et al., 2014; Sidhu et al., 2017). However, a greater recovery of maximal sprint power after incremental cycling to exhaustion with $60 \mathrm{~s}$ compared to $10 \mathrm{~s}$ post-exercise occlusion suggests group III/IV afferent stimulation has little impact on human endurance performance (Torres-Peralta et al., 2015). Whilst we cannot confirm the group III/IV muscle afferent hypothesis as a mechanisms, the data here show that training under high compared with moderate levels of peripheral fatigue (O'Leary et al., 2016) increases peripheral fatigue which may contribute to increased post-rel endurance capacity. Indeed, a number of mechanisms are plausible including peripheral fatigue being less sensed or alterations in central processes independent of any peripheral adaptation, the latter which is partly supported by the central fatigue data.

Central and supraspinal fatigue was induced by all trials as measured by reductions in VA and cortical VA, respectively. Similar to peripheral fatigue, central or supraspinal fatigue were not different after the post-abs trials compared to pre-training. Whilst there was also no effect of CONT on central or supraspinal fatigue following the post-rel trial, HIIT attenuated central fatigue. There were also moderate effect sizes for HIIT attenuating supraspinal fatigue after the post-rel trial $(\mathrm{D}=0.53)$, with a large effect size for a difference between groups $\left(\eta_{\mathrm{p}}{ }^{2}=0.17\right)$, and the lack of a significant effect $(\mathrm{P}=0.089)$ is therefore likely due to the small sample size. The contributions of central and peripheral mechanisms to fatigue are exercise duration dependent with longer duration exercise resulting in a greater central fatigue 
component (Thomas et al., 2015; Thomas et al., 2016). We however report less central fatigue following the post-rel trial after HIIT compared to pre-training, despite an increase in exercise duration. Therefore, the attenuation of central fatigue is a training adaptation and not due to differences in exercise duration.

In contrast, Zghal et al., (2015) reported that low force knee extensor training had no effect on the degree of central and supraspinal fatigue at exhaustion following the low force isometric endurance test, although a trend for more central fatigue post-training was noted. Differences between studies is likely due to differences in the mode of exercise. Consequently, this is the first study to demonstrate that HIIT develops central fatigue resistance during high-intensity exhaustive whole-body locomotor exercise. A number of CNS adaptations could contribute to central fatigue resistance such as improved handling of serotonin (Jakeman et al. 1994), attenuated cerebral ammonia uptake (Nybo et al. 2005), improved cerebral oxygenation (Rooks et al. 2010) and enhanced spinal reflex excitability (Vila-Cha et al. 2012). Zghal et al., (2015) recently hypothesised that training increases resistance to the central inhibitory effects of group III/IV afferent firing. As previously discussed, although group III/IV muscle afferents may play a role in the development on central fatigue (Amann et al., 2015), their effect on human performance is unclear (TorresPeralta et al., 2015). As CONT did not develop central fatigue resistance, it is therefore possible that HIIT may develop a resistance to the inhibitory group III/IV afferent feedback or result in these afferents being less stimulated in response to the same exercise challenge, allowing greater performance to be extruded from the muscle. Whilst the increase peripheral fatigue supports this, the underlying mechanism cannot be confirmed from the methodology employed here and there is currently a lack of evidence to support this hypothesis. 


\section{Limitations}

The results from this study should be considered alongside the following limitations. Firstly, measurements of fatigue were made post-exercise and may not translate to mechanisms during exercise. This is a general limitation with locomotor exercise and fatigue studies and the measurement protocol was identical between pre- and post-training trials as well as between training groups. We can therefore be confident that differences between pre- and post-training as well as between groups was due to a training effect. This is further supported by previous demonstration of the repeatability of these measures after exhaustive exercise and sensitivity in detecting differences between exercise intensities (O'Leary et al., 2016). Our sample size may have also been insufficient to detect some subtle differences between training groups such as changes in the level of supraspinal fatigue. Finally, we included both men and women in this study and a number of sex differences in the neuromuscular responses to exercise have been demonstrated (Hunter, 2016). Whilst the training groups were matched for numbers of men and women, and therefore any effect of sex differences in fatigability are well controlled for between groups, it is unclear whether men and women responded differently to each training intervention, which is an area warranting further investigation.

\section{Perspectives}

The findings from this study demonstrate that HIIT attenuates central fatigue and increases peripheral fatigue following exhaustive high-intensity exercise. This suggests that the development of central fatigue resistance allows greater performance to be extruded from the muscle in order to continue exercise. Group III/IV afferents have been proposed as an important contributor to fatigue and exhaustion, and HIIT may increase resistance to the inhibitory effects of these afferents or result in these afferents being less stimulated, however 
this is yet to be confirmed. Additionally, HIIT may also increase the ability to tolerate the physiological disturbance, high levels of effort or muscle pain experienced during highintensity exercise. These observations help to provide mechanistic insight into the mechanisms that limit endurance capacity and the fatigue adaptations induced by endurance training.

\section{Acknowledgements}

The authors wish to express gratitude to all research participants who took part in the study.

\section{Conflicts of Interest}

The authors declare no conflicts of interest.

\section{References}

Allen DG, Lamb GD, Westerblad H. Skeletal muscle fatigue: cellular mechanisms. Physiol Rev 2008: 88: 287-332.

Amann M. Central and peripheral fatigue: interaction during cycling exercise in humans. Med Sci Sports Exerc 2011: 43: 2039-2045.

Amann M, Sidhu SK, Weavil JC, Mangum TS, Venturelli M. Autonomic responses to exercise: group III/IV muscle afferents and fatigue. Auton Neurosci 2015: 188: 19-23.

Burgomaster KA, Hughes SC, Heigenhauser GJ, Bradwell SN, Gibala MJ. Six sessions of sprint interval training increases muscle oxidative potential and cycle endurance capacity in humans. J Appl Physiol 2005: 98: 1985-1990.

Chen R, Cros D, Curra A, Di Lazzaro V, Lefaucheur JP, Magistris MR, Mills K, Rosler KM, Triggs WJ, Ugawa Y, Ziemann U. The clinical diagnostic utility of transcranial magnetic stimulation: report of an IFCN committee. Clin Neurophysiol 2008: 119: 504-532. 
Daussin FN, Zoll J, Dufour SP, Ponsot E, Lonsdorfer-Wolf E, Doutreleau S, Mettauer B, Piquard F, Geny B, Richard R. Effect of interval versus continuous training on cardiorespiratory and mitochondrial functions: relationship to aerobic performance improvements in sedentary subjects. Am J Physiol Regul Integr Comp Physiol 2008: 295: R264-272.

Decorte N, Lafaix PA, Millet GY, Wuyam B, Verges S. Central and peripheral fatigue kinetics during exhaustive constant-load cycling. Scand J Med Sci Sports 2012: 22: 381-391.

Demarle AP, Slawinski JJ, Laffite LP, Bocquet VG, Koralsztein JP, Billat VL. Decrease of $\mathrm{O}_{2}$ deficit is a potential factor in increased time to exhaustion after specific endurance training. J Appl Physiol 2001: 90: 947-953.

Edge J, Bishop D, Goodman C. The effects of training intensity on muscle buffer capacity in females. Eur J Appl Physiol 2006: 96: 97-105.

Gandevia SC. Spinal and supraspinal factors in human muscle fatigue. Physiol Rev 2001: 81: $1725-1789$.

Gandevia SC, Allen GM, Butler JE, Taylor JL. Supraspinal factors in human muscle fatigue: evidence for suboptimal output from the motor cortex. J Physiol 1996: 490: 529-536.

Goodall S, Charlton K, Hignett C, Prichard J, Barwood M, Howatson G, Thomas K. Augmented supraspinal fatigue following constant-load cycling in the heat. Scand J Med Sci Sports 2015: 25 Suppl 1: 164-172.

Goodall S, Gonzalez-Alonso J, Ali L, Ross EZ, Romer LM. Supraspinal fatigue after normoxic and hypoxic exercise in humans. J Physiol 2012: 590: 2767-2782.

Goodall S, Romer LM, Ross EZ. Voluntary activation of human knee extensors measured using transcranial magnetic stimulation. Exp Physiol 2009: 94: 995-1004.

Hunter SK. The relevance of sex differences in performance fatigability. Med Sci Sports Exerc 2016: 48: 2241-2256. 
Jacobs RA, Fluck D, Bonne TC, Burgi S, Christensen PM, Toigo M, Lundby C. Improvements in exercise performance with high-intensity interval training coincide with an increase in skeletal muscle mitochondrial content and function. J Appl Physiol 2013: 115: 785-793.

Jakeman PM, Hawthorne JE, Maxwell SR, Kendall MJ, Holder G. Evidence for downregulation of hypothalamic 5-hydroxytryptamine receptor function in endurance-trained athletes. Exp Physiol 1994: 79: 461-464.

Jones AM, Carter H. The effect of endurance training on parameters of aerobic fitness. Sports Med 2000: 29: 373-386.

MacInnis MJ, Gibala MJ. Physiological adaptations to interval training and the role of exercise intensity. J Physiol 2016 [epub ahead of print].

Mann T, Lamberts RP, Lambert MI. Methods of prescribing relative exercise intensity: physiological and practical considerations. Sports Med 2013: 43: 613-625.

Merton PA. Voluntary strength and fatigue. J Physiol 1954: 123: 553-564.

Millet GY. Can neuromuscular fatigue explain running strategies and performance in ultramarathons?: the flush model. Sports Med 2011: 41: 489-506.

Morales-Alamo D, Losa-Reyna J, Torres-Peralta R, Martin-Rincon M, Perez-Valera M, Curtelin D, Ponce-Gonzalez JG, Santana A, Calbet JA. What limits performance during whole-body incremental exercise to exhaustion in humans? J Physiol 2015: 593: 4631-4648. Nybo L, Dalsgaard MK, Steensberg A, Moller K, Secher NH. Cerebral ammonia uptake and accumulation during prolonged exercise in humans. J Physiol 2005: 563: 285-290.

Nybo L, Secher NH. Cerebral perturbations provoked by prolonged exercise. Prog Neurobiol 2004: 72: 223-261. 
O'Leary TJ, Morris MG, Collett J, Howells K. Reliability of single and paired-pulse transcranial magnetic stimulation in the vastus lateralis muscle. Muscle Nerve 2015: 52: 605615.

O'Leary TJ, Morris MG, Collett J, Howells K. Central and peripheral fatigue following nonexhaustive and exhaustive exercise of disparate metabolic demands. Scand J Med Sci Sports 2016: 26: 1287-1300.

Poole DC, Gaesser GA. Response of ventilatory and lactate thresholds to continuous and interval training. J Appl Physiol 1985: 58: 1115-1121.

Pringle JS, Jones AM. Maximal lactate steady state, critical power and EMG during cycling. Eur J Appl Physiol 2002: 88: 214-226.

Rooks CR, Thom NJ, McCully KK, Dishman RK. Effects of incremental exercise on cerebral oxygenation measured by near-infrared spectroscopy: a systematic review. Prog Neurobiol 2010: 92: 134-150.

Seiler S, Joranson K, Olesen BV, Hetlelid KJ. Adaptations to aerobic interval training: interactive effects of exercise intensity and total work duration. Scand J Med Sci Sports 2013: 23: $74-83$

Sidhu SK, Bentley DJ, Carroll TJ. Cortical voluntary activation of the human knee extensors can be reliably estimated using transcranial magnetic stimulation. Muscle Nerve 2009: 39: 186-196.

Sidhu SK, Bentley DJ, Carroll TJ. Locomotor exercise induces long-lasting impairments in the capacity of the human motor cortex to voluntarily activate knee extensor muscles. J Appl Physiol 2009: 106: 556-565.

Sidhu SK, Cresswell AG, Carroll TJ. Corticospinal responses to sustained locomotor exercises: moving beyond single-joint studies of central fatigue. Sports Med 2013: 43: 437449. 
Sidhu SK, Weavil JC, Mangum TS, Jessop JE, Richardson RS, Morgan DE, Amann M. Group III/IV locomotor muscle afferents alter motor cortical and corticospinal excitability and promote central fatigue during cycling exercise. Clin Neurophysiol 2017: 128: 44-55.

Sidhu SK, Weavil JC, Venturelli M, Garten RS, Rossman MJ, Richardson RS, Gmelch BS, Morgan DE, Amann M. Spinal $\mu$-opioid receptor-sensitive lower limb muscle afferents determine corticospinal responsiveness and promote central fatigue in upper limb muscle. $\mathbf{J}$ Physiol 2014: 592: 5011-5024.

Thomas K, Elmeua M, Howatson G, Goodall S. Intensity-dependent contribution of neuromuscular fatigue after constant-load cycling. Med Sci Sports Exerc 2016: 48: 17511760.

Thomas K, Goodall S, Stone M, Howatson G, Gibson AS, Ansley L. Central and peripheral fatigue in male cyclists after 4-, 20-, and 40-km time trials. Med Sci Sports Exerc 2015: 47: 537-546.

Torres-Peralta R, Morales-Alamo D, Gonzalez-Izal M, Losa-Reyna J, Perez-Suarez I, Izquierdo M, Calbet JA. Task failure during exercise to exhaustion in normoxia and hypoxia is due to reduced muscle activation caused by central mechanisms while muscle metaboreflex does not limit performance. Front Physiol 2015: 6: 414.

Tschakert G, Hofmann P. High-intensity intermittent exercise: methodological and physiological aspects. Int J Sports Physiol Perform 2013: 8: 600-610.

Vila-Cha C, Falla D, Correia MV, Farina D. Changes in H reflex and V wave following short-term endurance and strength training. J Appl Physiol 2012: 112: 54-63.

Weston AR, Myburgh KH, Lindsay FH, Dennis SC, Noakes TD, Hawley JA. Skeletal muscle buffering capacity and endurance performance after high-intensity interval training by welltrained cyclists. Eur J Appl Physiol 1997: 75: 7-13. 
Zghal F, Cottin F, Kenoun I, Rebai H, Moalla W, Dogui M, Tabka Z, Martin V. Improved tolerance of peripheral fatigue by the central nervous system after endurance training. Eur $\mathrm{J}$ Appl Physiol 2015: 115: 1401-1415. 


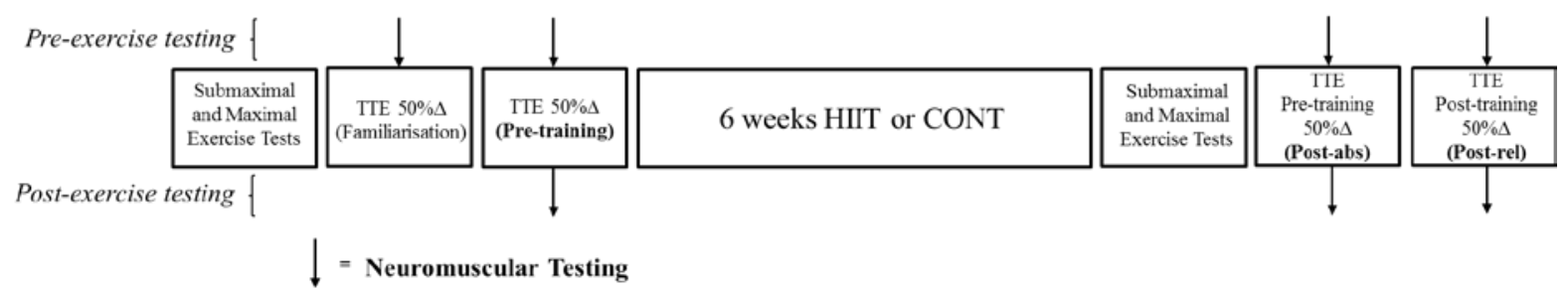

b

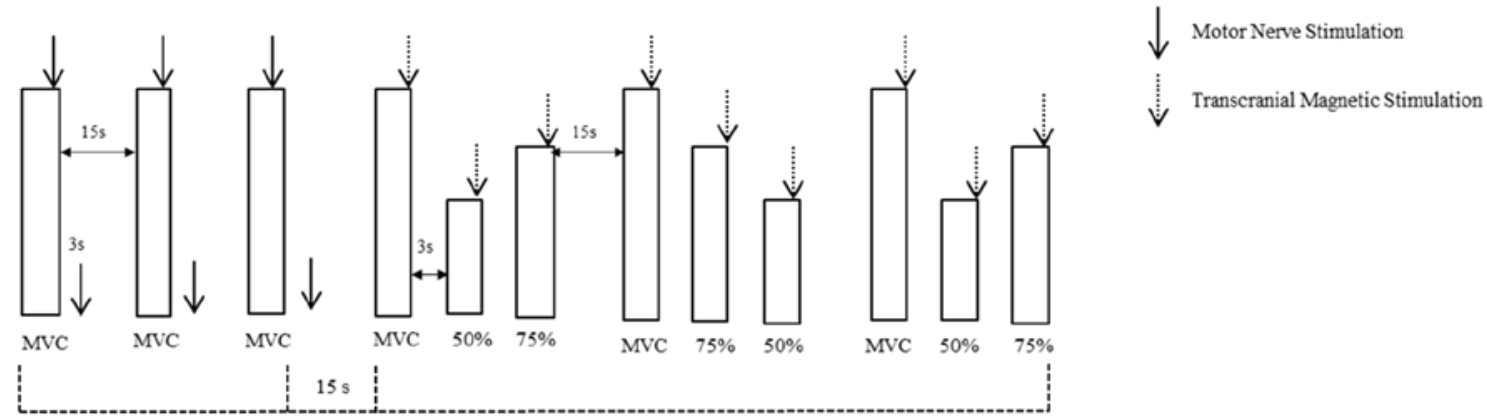

Fig. 1. Overview of the study design (a) and neuromuscular testing protocol (b). a) Two time to exhaustion (TTE) trials at an intensity equivalent to halfway between the lactate threshold and maximal oxygen uptake $(50 \% \Delta)$ were completed pre- and post- 6 weeks of high-intensity interval training (HIIT) or moderate-intensity continuous training (CONT). The first of the two post-training TTE trials was completed at the same power output as the pre-training $50 \% \Delta$ (same absolute intensity [post-abs]) and the second TTE trials was completed at the 'new' post-training 50\% $\Delta$ intensity (same relative intensity [post-rel]). b) Neuromuscular function was examined using motor nerve stimulation and transcranial magnetic stimulation pre- and post-exercise to examine the effect of training on central and peripheral fatigue development.

CONT, moderate-intensity continuous training; HIIT, high-intensity interval training; post-abs, same absolute intensity as pre-training; post-rel, same relative intensity as pre-training; TTE, time to exhaustion 


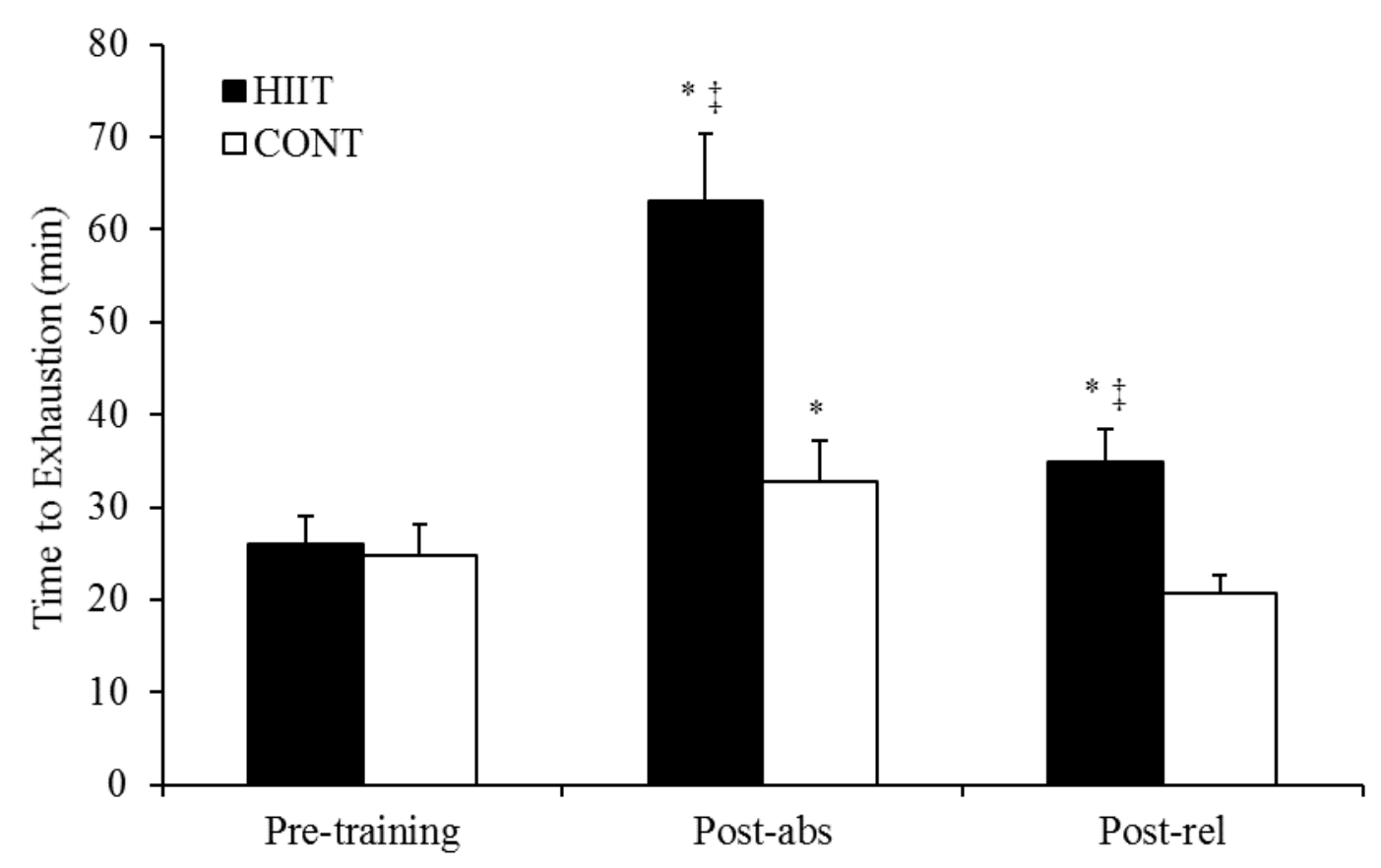

Fig. 2. Endurance capacity (time to exhaustion) before (pre-training) and after (post-abs and post-rel) 6 weeks of HIIT (filled bars) or CONT (hollow bars). Time to exhaustion tests were completed at an intensity equivalent to either the pre-training (pre-training and post-abs) or post-training (post-rel) $50 \% \Delta$. Data are mean $\pm \mathrm{SE}$

Abbreviations as in Fig. 1

*P $<0.05$ vs pre-training; ${ }^{*} \mathrm{P}<0.05$ vs CONT 


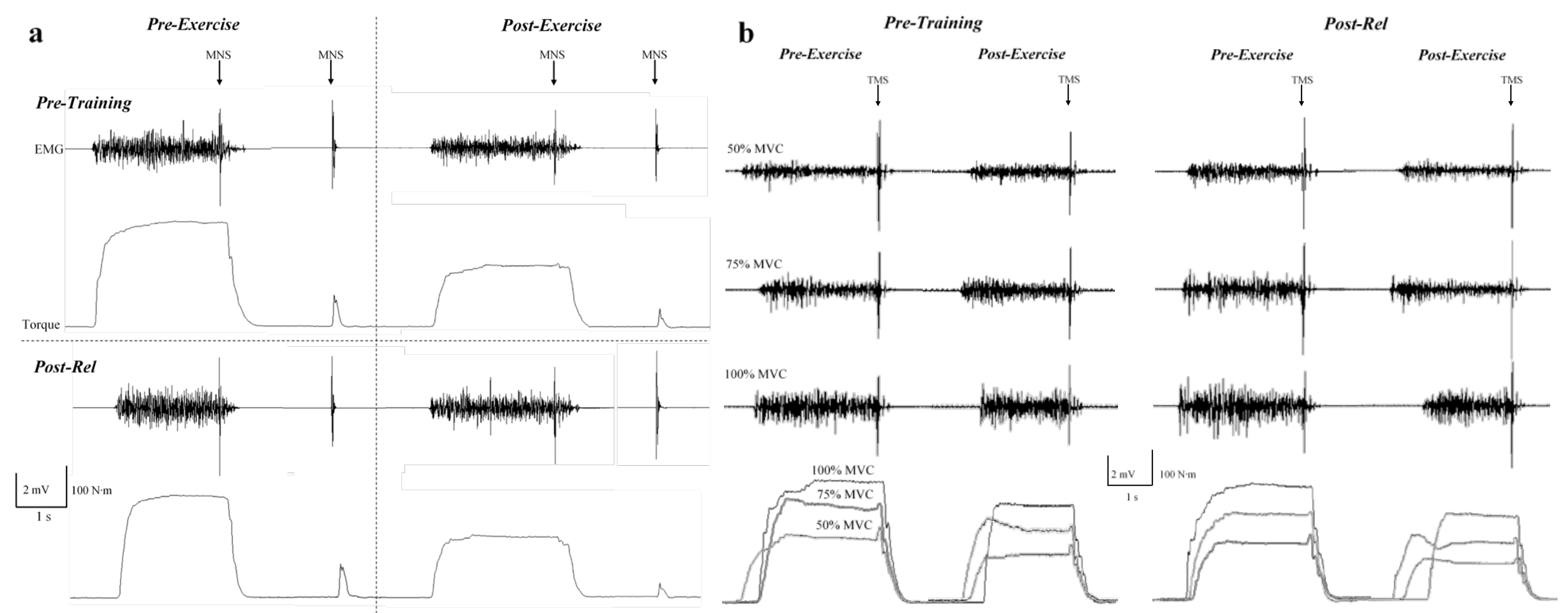

Fig. 3. Typical electromyography and torque traces in response to motor nerve stimulation (a) and transcranial magnetic stimulation (b) for the measurement of neuromuscular fatigue. Data are for a representative participant before (pre-training) and after (post-relative) high-intensity interval training. 


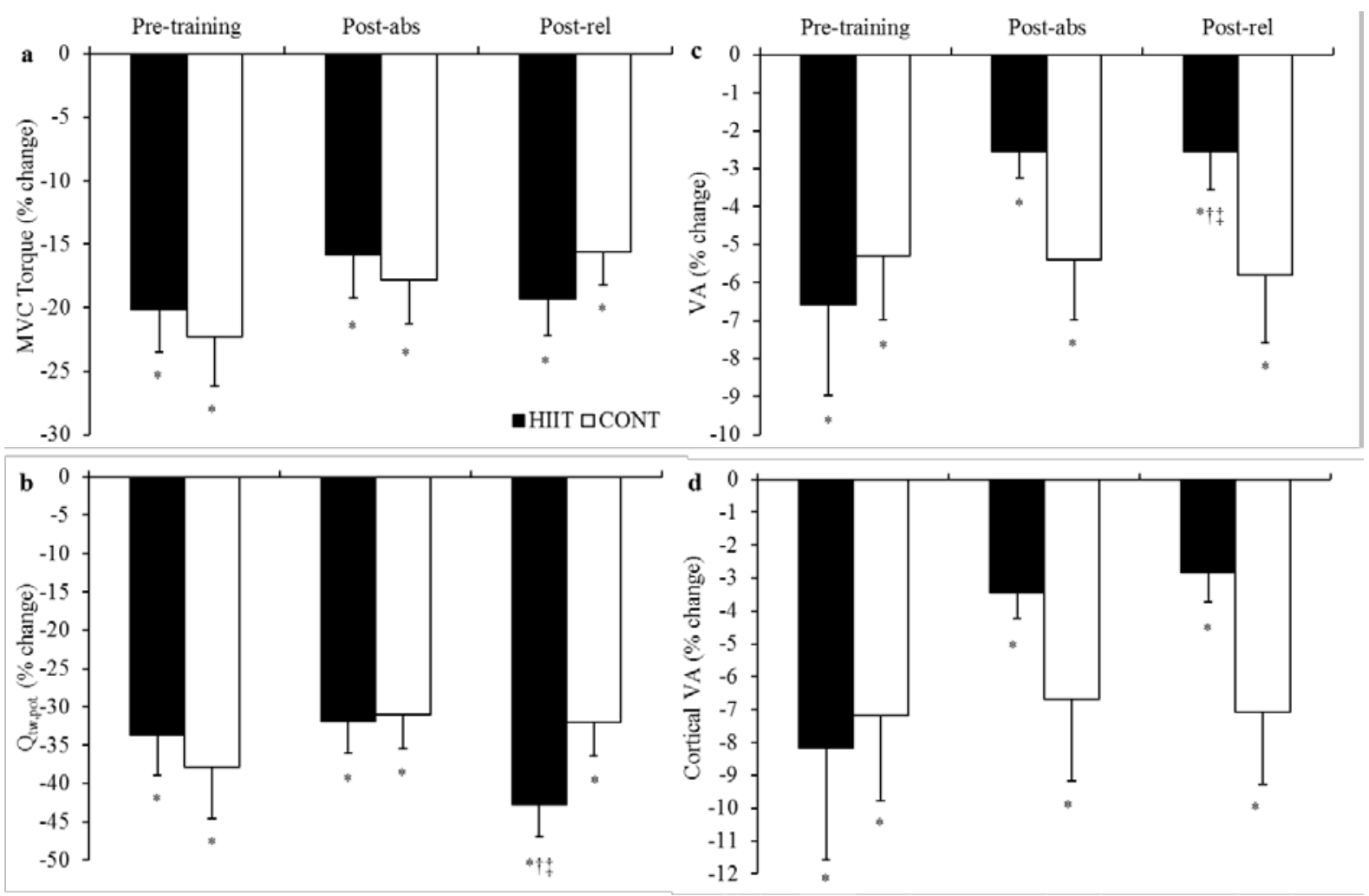

Fig. 4. Peripheral and central fatigue in response to the endurance capacity trials before (pretraining) and after (post-abs and post-rel) 6 weeks of HIIT (filled bars) or CONT (hollow bars). a, maximal voluntary torque; b, potentiated quadriceps twitch torque; c, voluntary activation; d, cortical voluntary activation. Data are relative change (\%) from pre-exercise $($ mean $\pm \mathrm{SE})$.

Abbreviations as in Fig. 1

${ }^{*} \mathrm{P}<0.05$ vs pre-exercise; ${ }^{\dagger} \mathrm{P}<0.05$ vs pre-training; ${ }^{\dagger} \mathrm{P}<0.05$ vs CONT 
Table 1. Characteristics of the HIIT and CONT protocols. Data are mean $\pm \mathrm{SD}$.

\begin{tabular}{|c|c|c|}
\hline & HIIT & CONT \\
\hline \multicolumn{3}{|l|}{ First Session } \\
\hline Power Output (Watts) & $202 \pm 47^{\dagger}$ & $103 \pm 40$ \\
\hline$\%$ pre-training $\dot{V} O_{2 \max }$ & $80 \pm 6^{\dagger}$ & $50 \pm 6$ \\
\hline Protocol & $6 \times 5$ min with 1 min rest & $58.8^{\circ} \pm 9.8$ min continuous \\
\hline Total Work (kJ) & $363 \pm 84$ & $346 \pm 102$ \\
\hline Mean HR $\left(b \cdot \mathrm{min}^{-1}\right)$ & $164 \pm 7^{\dagger}$ & $125 \pm 12$ \\
\hline Mean $H R\left(\% H R_{\max }\right)$ & $89 \pm 5^{\dagger}$ & $67 \pm 4$ \\
\hline Mean RPE (6-20) & $16 \pm 1^{\dagger}$ & $12 \pm 1$ \\
\hline \multicolumn{3}{|l|}{ Last Session } \\
\hline Power Output (Watts) & $228 \pm 45^{* \dagger}$ & $129 \pm 42 *$ \\
\hline Protocol & $8 \times 5$ min with 1 min rest & $77.7 \pm 13.7$ min continuous \\
\hline Total Work (kJ) & $545 \pm 103 *$ & $562 \pm 148^{*}$ \\
\hline Mean HR $\left(b \cdot \mathrm{min}^{-1}\right)$ & $164 \pm 9^{\dagger}$ & $128 \pm 11$ \\
\hline Mean $H R\left(\% H R_{\max }\right)$ & $89 \pm 6^{\dagger}$ & $67 \pm 5$ \\
\hline Mean RPE (6-20) & $16 \pm 2^{\dagger}$ & $12 \pm 2$ \\
\hline No. of Sessions & $18 \pm 1$ & $18 \pm 1$ \\
\hline Total Volume (kJ) & $7918 \pm 1541$ & $8105 \pm 2036$ \\
\hline
\end{tabular}

*P $<0.05$ vs pre-training; ${ }^{\dagger} \mathrm{P}<0.05$ vs CONT. 
Table 2. Aerobic fitness before and after 6 weeks HIIT or CONT. Data are mean \pm SD.

\begin{tabular}{|c|c|c|c|c|c|}
\hline & \multicolumn{2}{|c|}{ HIIT } & \multicolumn{2}{|c|}{ CONT } & \multirow{2}{*}{$\begin{array}{c}\text { Effect Size }^{7} \\
\eta_{\mathrm{p}}{ }^{2}\end{array}$} \\
\hline & Pre-training & $\begin{array}{c}\text { Post- } \\
\text { training }\end{array}$ & Pre-training & $\begin{array}{c}\text { Post- } \\
\text { training }\end{array}$ & \\
\hline \multicolumn{6}{|l|}{ LT } \\
\hline Watts & $112 \pm 36$ & $138 \pm 43^{*}$ & $106 \pm 37$ & $128 \pm 39 *$ & 0.022 \\
\hline$\% \dot{V} O_{2 \max }$ & $52 \pm 8$ & $56 \pm 10^{*}$ & $52 \pm 9$ & $55 \pm 8^{*}$ & 0.001 \\
\hline$\left[\mathrm{La}^{-}\right]\left(\mathrm{mmol} \cdot \mathrm{l}^{-1}\right)$ & $1.5 \pm 0.2$ & $1.3 \pm 0.3$ & $1.4 \pm 0.4$ & $1.3 \pm 0.2$ & -- \\
\hline \multicolumn{6}{|l|}{ LTP } \\
\hline Watts & $158 \pm 38$ & $186 \pm 35^{*}$ & $158 \pm 38$ & $174 \pm 40^{*}$ & 0.087 \\
\hline$\% \dot{V} O_{2 \max }$ & $68 \pm 8$ & $72 \pm 8^{*}$ & $68 \pm 7$ & $70 \pm 8^{*}$ & 0.018 \\
\hline$\left[\mathrm{La}^{-}\right]\left(\mathrm{mmol} \cdot \mathrm{l}^{-1}\right)$ & $3.3 \pm 0.7$ & $3.2 \pm 0.7$ & $3.4 \pm 0.8$ & $3.4 \pm 0.8$ & -- \\
\hline \multicolumn{6}{|l|}{$\dot{\mathbf{V}} \mathbf{O}_{2 \max }$} \\
\hline$l \cdot \min ^{-1}$ & $3.52 \pm 0.71$ & $3.80 \pm 0.75^{*}$ & $3.33 \pm 0.92$ & $3.62 \pm 0.99^{*}$ & 0.000 \\
\hline \multicolumn{6}{|l|}{$50 \% \Delta$} \\
\hline Watts & $202 \pm 47$ & $223 \pm 50^{*}$ & $189 \pm 52$ & $201 \pm 51^{*}$ & 0.115 \\
\hline$\% \dot{V} O_{2 \max }$ & $80 \pm 6$ & $80 \pm 5$ & $79 \pm 5$ & $78 \pm 6$ & 0.001 \\
\hline
\end{tabular}

[La'], capillary lactate concentrations; LT, lactate threshold; LTP, lactate turnpoint; $\dot{\mathrm{VO}}_{2 \max }$, maximal oxygen uptake; $50 \% \Delta$, exercise intensity halfway between lactate threshold and maxima oxygen uptake.

$* \mathrm{P}<0.05$ vs pre-training; ${ }^{*}$ group $\times$ time interaction. 
Table 3. Physiological and perceptual responses during the endurance capacity trials before (pre-training) and after (post-abs and post-rel) 6 weeks of HIIT or CONT. Data are mean \pm SD.

\begin{tabular}{|c|c|c|c|c|c|c|c|c|c|}
\hline & \multicolumn{3}{|c|}{ Pre-training } & \multicolumn{3}{|c|}{ Post-abs } & \multicolumn{3}{|c|}{ Post-rel } \\
\hline & Rest & $10 \mathrm{~min}$ & Last min & Rest & $10 \mathrm{~min}$ & Last min & Rest & $10 \mathrm{~min}$ & Last min \\
\hline \multicolumn{10}{|c|}{ HR $\left(b \cdot \min ^{-1}\right)$} \\
\hline HIIT & $74 \pm 13$ & $164 \pm 7$ & $171 \pm 12$ & $74 \pm 12$ & $157 \pm 11^{*}$ & $170 \pm 10$ & $72 \pm 17$ & $163 \pm 10$ & $172 \pm 11$ \\
\hline CONT & $76 \pm 12$ & $168 \pm 14$ & $174 \pm 12$ & $76 \pm 9$ & $164 \pm 14$ & $173 \pm 11$ & $77 \pm 10$ & $170 \pm 11$ & $174 \pm 10$ \\
\hline \multicolumn{10}{|c|}{$\dot{\mathrm{V}} \mathrm{O}_{2}\left(l \cdot \mathrm{min}^{-1}\right)$} \\
\hline HIIT & $0.44 \pm 0.08$ & $2.96 \pm 0.73$ & $3.14 \pm 0.71$ & $0.46 \pm 0.15$ & $2.85 \pm 0.61$ & $3.07 \pm 0.65$ & $0.44 \pm 0.07$ & $3.20 \pm 0.71 *$ & $3.40 \pm 0.78 *$ \\
\hline CONT & $0.40 \pm 0.07$ & $2.75 \pm 0.71$ & $3.00 \pm 0.66$ & $0.40 \pm 0.16$ & $2.65 \pm 0.80$ & $2.93 \pm 0.78$ & $0.41 \pm 0.16$ & $2.81 \pm 0.76$ & $3.09 \pm 0.72$ \\
\hline \multicolumn{10}{|c|}{$\dot{\mathbf{V}} \mathbf{O}_{2}\left(\% \dot{\boldsymbol{V}} \boldsymbol{O}_{2 \max }\right)$} \\
\hline HIIT & $13 \pm 1$ & $83 \pm 7$ & $88 \pm 7$ & $12 \pm 3$ & $75 \pm 7 *$ & $82 \pm 6^{*}$ & $12 \pm 2$ & $84 \pm 9$ & $90 \pm 9$ \\
\hline CONT & $13 \pm 3$ & $82 \pm 8$ & $86 \pm 9$ & $11 \pm 3$ & $73 \pm 9 *$ & $77 \pm 10^{*}$ & $12 \pm 3$ & $78 \pm 7$ & $87 \pm 11$ \\
\hline \multicolumn{10}{|l|}{ RER } \\
\hline HIIT & $0.95 \pm 0.04$ & $1.13 \pm 0.07$ & $1.13 \pm 0.09$ & $0.97 \pm 0.09$ & $1.06 \pm 0.06^{*}$ & $1.11 \pm 0.08$ & $0.98 \pm 0.11$ & $1.12 \pm 0.08$ & $1.14 \pm 0.09$ \\
\hline CONT & $0.96 \pm 0.11$ & $1.14 \pm 0.09$ & $1.13 \pm 0.09$ & $0.97 \pm 0.05$ & $1.12 \pm 0.06$ & $1.13 \pm 0.09$ & $0.95 \pm 0.07$ & $1.15 \pm 0.10$ & $1.15 \pm 0.10$ \\
\hline \multicolumn{10}{|c|}{$\left[\mathrm{La}^{-}\right]\left(\mathrm{mmol}^{\prime} \cdot \mathrm{l}^{1}\right)$} \\
\hline HIIT & $1.4 \pm 0.5$ & $6.8 \pm 1.9$ & $8.6 \pm 3.3$ & $1.3 \pm 0.4$ & $4.1 \pm 1.3^{*}$ & $5.8 \pm 2.4^{*}$ & $1.4 \pm 0.3$ & $6.2 \pm 2.0$ & $8.8 \pm 2.8$ \\
\hline CONT & $1.3 \pm 0.3$ & $6.8 \pm 2.1$ & $7.6 \pm 2.8$ & $1.2 \pm 0.4$ & $5.3 \pm 1.8^{*}$ & $5.8 \pm 2.8^{*}$ & $1.3 \pm 0.4$ & $6.2 \pm 1.1$ & $7.2 \pm 1.0$ \\
\hline \multicolumn{10}{|c|}{ RPE (6-20) } \\
\hline HIIT & $6 \pm 0$ & $16 \pm 2$ & $20 \pm 1$ & $6 \pm 0$ & $13 \pm 2 *$ & $20 \pm 1$ & $6 \pm 0$ & $15 \pm 2$ & $20 \pm 1$ \\
\hline CONT & $6 \pm 0$ & $16 \pm 2$ & $20 \pm 1$ & $6 \pm 0$ & $15 \pm 3$ & $20 \pm 0$ & $6 \pm 0$ & $17 \pm 2$ & $20 \pm 0$ \\
\hline
\end{tabular}

$\mathrm{HR}$, heart rate; [La-], capillary lactate concentration; RER, respiratory exchange ratio; RPE, ratings of perceived exertion; $\dot{\mathrm{VO}}_{2}$, oxygen uptake. $* \mathrm{P}<0.05$ vs pre-training; ${ }^{\dagger} \mathrm{P}<0.05$ vs CONT. 
Table 4. Neuromuscular responses to the endurance capacity trials before (pre-training) and after (post-abs and post-rel) 6 weeks of HIIT or CONT. Data are mean \pm SD.

\begin{tabular}{|c|c|c|c|c|c|c|c|c|c|}
\hline & \multicolumn{3}{|c|}{ Pre-training } & \multicolumn{3}{|c|}{ Post-abs } & \multicolumn{3}{|c|}{ Post-rel } \\
\hline & Pre- & Post- & Change (\%) & Pre- & Post- & Change (\%) & Pre- & Post- & Change (\%) \\
\hline \multicolumn{10}{|c|}{$\operatorname{MVC}(\mathbf{N} \cdot \mathbf{m})$} \\
\hline HIIT & $460 \pm 120$ & $365 \pm 103^{*}$ & -20 & $453 \pm 110$ & $377 \pm 90^{*}$ & -16 & $472 \pm 102$ & $380 \pm 90^{*}$ & -19 \\
\hline CONT & $388 \pm 120$ & $300 \pm 100^{*}$ & -22 & $391 \pm 123$ & $316 \pm 93^{*}$ & -18 & $393 \pm 119$ & $329 \pm 97 *$ & -16 \\
\hline \multicolumn{10}{|c|}{$M_{\max }(\mathrm{mV})$} \\
\hline HIIT & $6.57 \pm 1.33$ & $6.56 \pm 1.74$ & -1 & $6.79 \pm 1.40$ & $6.41 \pm 1.29$ & 0 & $6.91 \pm 1.15$ & $6.96 \pm 1.28$ & +1 \\
\hline CONT & $6.20 \pm 1.09$ & $5.79 \pm 1.13$ & -6 & $6.59 \pm 0.83$ & $6.53 \pm 1.15$ & 0 & $5.84 \pm 1.24$ & $5.81 \pm 1.50$ & 0 \\
\hline \multicolumn{10}{|c|}{$\mathbf{Q}_{\mathrm{tw}, \mathrm{pot}}(\mathbf{N} \cdot \mathbf{m})$} \\
\hline HIIT & $126 \pm 37$ & $83 \pm 29^{*}$ & -34 & $132 \pm 29$ & $91 \pm 32 *$ & -32 & $141 \pm 29$ & $80 \pm 30 * \dagger$ & $-43^{\#}$ \\
\hline CONT & $111 \pm 34$ & $70 \pm 36^{*}$ & -38 & $119 \pm 26$ & $82 \pm 26^{*}$ & -31 & $117 \pm 29$ & $80 \pm 30^{*}$ & -32 \\
\hline \multicolumn{10}{|c|}{ VA (\%) } \\
\hline HIIT & $97.8 \pm 1.2$ & $91.4 \pm 7.0^{*}$ & -7 & $98.0 \pm 1.1$ & $95.8 \pm 2.2^{*}$ & -3 & $97.9 \pm 1.4$ & $95.5 \pm 3.3^{* \dagger}$ & $-3^{\#}$ \\
\hline CONT & $94.9 \pm 6.4$ & $89.1 \pm 13.3^{*}$ & -5 & $95.9 \pm 6.5$ & $88.1 \pm 15.2 *$ & -5 & $95.9 \pm 6.5$ & $90.7 \pm 10.4^{*}$ & -6 \\
\hline \multicolumn{10}{|c|}{ Cortical VA (\%) } \\
\hline HIIT & $96.5 \pm 3.7$ & $88.7 \pm 11.9^{*}$ & -8 & $96.9 \pm 3.0$ & $94.3 \pm 2.7^{*}$ & -3 & $96.4 \pm 2.9$ & $94.6 \pm 2.4^{*}$ & -3 \\
\hline CONT & $94.6 \pm 5.0$ & $87.8 \pm 9.2 *$ & -7 & $94.4 \pm 4.7$ & $88.0 \pm 8.6^{*}$ & -7 & $93.7 \pm 5.7$ & $87.0 \pm 8.0^{*}$ & -7 \\
\hline \multicolumn{10}{|c|}{ MEP/M $\mathbf{M}_{\text {Sup }}(\%)$} \\
\hline HIIT & $69 \pm 29$ & $70 \pm 27$ & +7 & $63 \pm 31$ & $60 \pm 27$ & 0 & $62 \pm 25$ & $57 \pm 24$ & -4 \\
\hline CONT & $65 \pm 21$ & $59 \pm 23$ & -10 & $60 \pm 24$ & $61 \pm 26$ & +5 & $61 \pm 27$ & $66+33$ & +11 \\
\hline
\end{tabular}

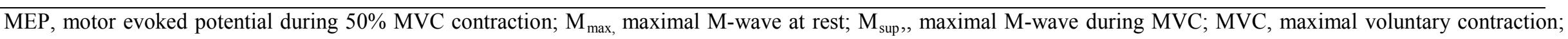
Pre-, pre-exercise; Post-, post-exercise; $\mathrm{Q}_{\mathrm{tw} \text {,pot, }}$ potentiated quadriceps twitch; VA, voluntary activation.

${ }^{*} \mathrm{P}<0.05$ vs pre-exercise; $\uparrow \mathrm{P}<0.05$ vs pre-training; ${ }^{\#} \mathrm{P}<0.05$ vs CONT. 